\title{
Standard of Practice for the Endovascular Treatment of Thoracic Aortic Aneurysms and Type B Dissections
}

\author{
Fabrizio Fanelli · Michael D. Dake
}

Received: 25 June 2009/ Accepted: 8 July 2009/Published online: 18 August 2009

(c) The Author(s) 2009. This article is published with open access at Springerlink.com

\begin{abstract}
Thoracic endovascular aortic repair (TEVAR) represents a minimally invasive technique alternative to conventional open surgical reconstruction for the treatment of thoracic aortic pathologies. Rapid advances in endovascular technology and procedural breakthroughs have contributed to a dramatic transformation of the entire field of thoracic aortic surgery. TEVAR procedures can be challenging and, at times, extraordinarily difficult. They require seasoned endovascular experience and refined skills. Of all endovascular procedures, meticulous assessment of anatomy and preoperative procedure planning are absolutely paramount to produce optimal outcomes. These guidelines are intended for use in quality-improvement programs that assess the standard of care expected from all physicians who perform TEVAR procedures.
\end{abstract}

This article is a CIRSE SOP document approved by the CIRSE Standard of Practice Committee and endorsed by the Cardiovascular Interventional Radiological Society of Europe.

F. Fanelli $(\bowtie)$

Department of Radiological Sciences,

Vascular and Interventional Radiology Unit,

"Sapienza," University of Rome, 324 Viale Regina Elena, 00161 Rome, Italy

e-mail: fabrizio.fanelli@uniroma1.it

M. D. Dake

Department of Cardiothoracic Surgery Radiology,

Stanford University School of Medicine,

Falk Cardiovascular Research Center, 300 Pasteur Drive,

Stanford, CA 94305-5407, USA
Keywords Aortic dissection - Thoracic aorta - Thoracic aortic aneurysm $\cdot$ Standard of practice $\cdot$ CIRSE

\section{Introduction}

Thoracic endovascular aortic repair (TEVAR) represents a minimally invasive alternative to conventional open surgical reconstruction for the treatment of thoracic aortic pathologies. It is a valid therapeutic option for the treatment of thoracic aortic aneurysms and thoracic aortic dissections by virtue of its lower mortality, morbidity, and paraplegia rates compared to open thoracic repair. Rapid advances in endovascular technology and procedural breakthroughs have contributed to a dramatic transformation of the entire field of thoracic aortic surgery $<15$ years after the first report of stent-graft repair of thoracic aortic aneurysms [1]. TEVAR procedures can be challenging and, at times, extraordinarily difficult. They require seasoned endovascular experience and refined skills similar to all endovascular procedures. In addition, meticulous assessment of anatomy and preoperative procedure planning are absolutely paramount to produce optimal outcomes.

These guidelines are intended for use in qualityimprovement programs that assess the standard of care expected of all physicians who perform TEVAR procedures. The issues addressed in this article include:

- disease/lesion definition, diagnosis, and symptoms;

- pretreatment evaluation;

- patient selection;

- procedure and technical aspects;

- follow-up;

- outcomes: technical success, clinical results, and complications based on the currently available data. 


\section{Definition, Diagnosis, and Symptoms}

\section{Definition}

\section{Aneurysm}

The permanent and irreversible dilation of an artery is called aneurysm. Conventionally, according to the definition of the Ad Hoc Committee on Reporting Standards of the Society for Vascular Surgery and the North American Chapter of the International Society for Cardiovascular Surgery, a transverse diameter exceeding at least $150 \%$ of the diameter of the remaining part of the artery can be judged "aneurysmatic" [2-4].

According to conventions proposed by these societies, thoracic aortic aneurysms can be divided into:

- aneurysms that exist at the level of the ascending aorta, arch, or descending aorta, or involving all three of these segments (thoracic)

- thoracoabdominal aneurysms, with involvement of both the descending aorta and the abdominal aorta.

Based on the extent of disease and in compliance with the Crawford and coworkers' classification, these can be categorized further [5].

- Type 1: involving the proximal half of the descending aorta with extension as far as the renal arteries.

- Type 2: stretching from the proximal half of the descending thoracic aorta to the intrarenal aorta.

- Type 3: extending from the distal half of the descending thoracic aorta to the abdominal aorta.

- Type 4: affecting most of the abdominal aorta, with proximal involvement above the renal arteries.

According to their shape, thoracic aneurysms can be divided into two types:

- Fusiform: These aneurysms typically involve all three layers of the aortic wall and, thus, are usually true aneurysms. The abnormal dilation is often along an extended section of the aorta and involves the entire circumference of the aorta. Generally, the weakened portion appears as a symmetrical bulge.

- Saccular: These look like a small blister or bleb on the side of the aorta and are asymmetrical. Typically they are pseudoaneurysms caused either by trauma such as a car accident or as the result of a penetrating aortic ulcer.

Thoracic aorta aneurysms have a mean growth rate of $0.42 \mathrm{~cm} /$ year at the level of the descending aorta and of $0.56 \mathrm{~cm} /$ year at the level of the aortic arch $[6,7]$.

The larger the diameter of a thoracic aortic aneurysm, the higher the annual rupture risk [8, 9]:
$>4 \mathrm{~cm}, 0.3 \%$;

$>5 \mathrm{~cm}, 1.7 \%$;

$>6 \mathrm{~cm}, 3.6 \%$.

A surgical or endovascular treatment is recommended when the diameter at the level of the ascending aorta exceeds $49 \mathrm{~mm}$ and when the caliber at the level of the arch and of the descending aorta the diameter is $>45 \mathrm{~mm}$ [10, 11]. Cambria et al. [9] reported a 52\% survival rate 2 years postdiagnosis and a $17 \%$ survival rate at 5 years in untreated patients. In patients diagnosed with Marfan syndrome, treatment is recommended if the aortic diameter is $>43 \mathrm{~mm}$ [12].

Idiopathic cystic medial degeneration, atherosclerosis, connective tissue disorders (Marfan syndrome, EhlersDanlos syndrome), trauma, infection of the aortic wall, and Takayasu's arteritis are the principal aortic pathologies associated with thoracic aneurysm [10-13].

\section{Dissection}

Aortic dissection is caused by the formation of a false channel within the aortic wall consequent to a disruption of the intimal lining. A dissection plane that separates the intima from the surrounding adventitia is created within the media over a variable length of the aorta. This produces a false lumen or a double-barreled aorta that can reduce blood flow to the major arteries arising from the aorta [14]. If the dissection involves the pericardial space, cardiac tamponade may result.

The most common site for the initiation of dissection when the ascending aorta is involved is within its first few centimeters. In fact, the entry tear for the process occurs within $10 \mathrm{~cm}$ of the aortic valve in $90 \%$ of cases. The second most frequent site for the initiating tear is just distal to the left subclavian artery (LSA) [15]. The dissection can proceed for a variable distance, usually in an antegrade direction but sometimes retrograde from the site of the intimal tear. However, in the majority of cases, the dissection affects the descending aorta, with the primary entry tear located at the level of the origin of the left subclavian artery in $40 \%[16,17]$. Between $5 \%$ and $10 \%$ of dissections do not have an obvious intimal tear and are often attributed to rupture of the aortic vasa vasorum as first described by Krukenberg in 1920 [16, 17].

According to the site of aortic involvement, aortic dissections can be divided into two groups (Stanford classification) [18].

- Type A: The dissection involves the ascending aorta and may extend distally to include the aortic arch and the descending aorta. The primary entry tear is typically located at the level of the ascending aorta or in the arch, 
but may be in the descending aortic segment and associated with retrograde propagation to affect the ascending aorta.

- Type B: The primary entry tear is typically after the origin of the left subclavian artery and the descending aorta and/or aortic arch are affected exclusively, without involvement of the ascending aorta. Uncommonly, the entry tear may be located in the arch, with or without extension to the descending segment, but not into the ascending aorta.

On the basis of the time from the onset of the initial symptoms, a dissection can be categorized as either acute ( $<14$ days) or chronic ( $>14$ days) [19].

Moreover, Crawford et al. divided chronic type B dissections into different categories [20].

- Type 1: The dissection involves the descending aorta up to the origin of the renal arteries

- Type 2: The descending aorta is dissected over its entire length and the abdominal aorta up to the iliac arteries is involved.

- Type 3: The dissection starts at the level of the midthird of the descending aorta and involves the entire abdominal aorta.

- Type 4: The whole abdominal aorta, below the diaphragm, is involved.

The main causes of dissection are cystic medial necrosis, atherosclerosis (occlusion of the vasa vasorum), connective tissue disorders (Marfan syndrome, Ehlers-Danlos syndrome), hypertension, metabolic disorders, pregnancy, crack cocaine use, and iatrogenic (arterial catheterization) [21].

Dissection can also be divided into uncomplicated and complicated disease. Complicated dissection consists of one or more of the following manifestations: rupture, imminent rupture, branch vessel involvement with malperfusion syndrome or persistent or worsening thoracic pain, drug-resistant hypertension, and false lumen aneurysm formation.

\section{Symptoms}

\section{Aneurysm}

When carotid or coronary pathology does not coexist, thoracic aorta aneurysms are generally silent and diagnosed by sheer chance. But if the maximum diameter of the aneurysm exceeds $70 \mathrm{~mm}$, symptoms that correlate with the compression of adjacent organs may be reported [7, 8, 22, 23].

- Ascending aortic aneurysms: These often provoke aortic root dilation and leakage of the aortic valve, with consequent shortness of breath and even heart failure when the incompetence is severe. A dull substernal pain that may radiate to the upper back can also be present.

- Aortic arch aneurysms: These may be associated with upper chest or interscapular back pain. When aortic arch aneurysms are large, both the esophagus and the airway can be compressed. Difficulty swallowing and/ or hoarseness are the initial symptoms.

- Descending thoracic aneurysms: These are mostly asymptomatic. They can occasionally cause back pain.

\section{Dissection}

When the initial tear and dissection occur, the symptoms are severe, with an abrupt onset of pain [24]. The sudden pain is generally located at the midsternum for dissection of the ascending aorta and in the interscapular region for descending thoracic aortic dissection. "Migratory pain" should be regarded suspiciously as a sign of dissection extension in an antegrade or retrograde direction [25]. Painless dissection has been described and usually occurs in the presence of an existing aneurysm, where the pain of a new dissection may not be differentiated from chronic aneurysm pain [26].

Depending on the evolution of the dissection process, symptoms can be complicated by hypotension, bradycardia, abdominal pain, and intestinal or inferior limb ischemia [26]. However, a differential diagnosis for chest pain should be considered, with myocardial ischemia, aortic aneurysm, acute aortic regurgitation, pericarditis, musculoskeletal pain, and pulmonary embolus entertained [27, 28].

\section{Diagnosis}

Both thoracic aortic aneurysm and dissection are diagnosed by the same spectrum of imaging evaluations: plain chest radiography, CT angiography (CTA), magnetic resonance angiography (MRA), conventional digital subtraction angiography (DSA), and transesophageal echocardiography (TEE) [29-31].

On $X$-ray, abnormal findings are evident in $88 \%$ of cases and include the following.

- Widened mediastinum (25\% of cases): differential diagnosis is tumor, adenophaty, lymphoma, and enlarged thyroid.

- Abnormal aortic knob contour (66\% of cases).

- Tracheal or esophageal deviation.

- Ring sign (dissection), with displacement of the aortic margin $>5 \mathrm{~cm}$ beyond the calcified aortic intima.

CTA: CTA is the best noninvasive alternative to DSA and is considered the gold standard and primary diagnostic modality today. With a single breath-hold acquisition, after 
contrast medium injection, it can evaluate the thoracic and abdominal aorta, supraaortic vessels, abdominal branches, and iliac-femoral axis. Images can be studied in the standard axial format, and after postprocessing multiplanar reconstructions using different algorithms (volume rendering [VR], maximum intensity projection [MIP], multiplanar reformation [MPR], shaded surface display [SSD], etc.) provide three-dimensional characterizations.

CTA evaluations can provide the following:

- diameter and morphology of the aorta;

- diameter and length of the proximal and distal necks;

- intimal flap anatomy, extent of the dissection, and true and false lumen morphologies;

- site of the primary entry tear;

- re-entry site(s);

- presence of thrombus or calcifications;

- patency of the abdominal branches;

- size, tortuosity, and disease status of iliac and femoral arteries.

Recently a new imaging modality, electrocardiogram (ECG)-gated CTA, has been introduced. The 3D volumetric data sets allow rotation of the aorta while viewing it in different phases of the cardiac cycle. This may improve diagnostic accuracy, as motion artifacts, often the cause of false-positive findings of a thoracic dissection, are minimal [32, 33].

MRA: MRA allows limiting the exposure to ionizing radiation and forgoing the use of noniodinated contrast media, which are preferable in those cases where multiple follow-up examinations are required and in patients with iodinated contrast allergies. MRA was once considered ideal for patients with renal failure, but after the discovery of nephrogenic systemic fibrosis in patients with renal dysfunction receiving intravenous gadolinium, the initial enthusiasm drastically decreased. MRA gives the same information that can be achieved using CTA. The introduction of axial images, acquired with "black-blood" sequences, allows a better evaluation of the aortic wall and of any dissection, ulcer, or thrombus accumulation. This sequence takes advantage of the lack of signal from blood flowing perpendicular to the imaging plane.

MRA does have some limits; it gives no information about the presence of calcium in the aorta or conduit arteries and cannot be performed in patients with old cardiac valve prostheses or pacemakers [31, 34].

DSA: DSA is still considered the definitive diagnostic test for thoracic pathology, but it clearly displays limitations compared to the more modern CTA and MRA. Principally, this is because of the complications associated with an invasive procedure not encountered with noninvasive imaging tests and its inability to evaluate the thrombus quantity, when present, along the aortic wall. DSA is usually performed before any interventional procedure and when CTA and MRA cannot answer all diagnostic doubts.

DSA is mandatory when patients exhibit ischemic problems with mesenteric, renal, or lower extremity malperfusion, because it provides the final diagnostic data that contribute to the determination of whether a therapeutic intervention is required and feasible. It also provides vital morphological and hemodynamic data to guide any endovascular treatment.

TEE: TEE can be quickly performed at the patient's bedside, in no more than $20 \mathrm{~min}$. It has a diagnostic sensitivity ranging from $98 \%$ to $100 \%$ for dissection and a technical suitability for patient application, ranging from $90 \%$ to $100 \%$ [35]. Moreover, it can be performed more than once, its cost is limited, and it is useful during the follow-up of patients who have undergone surgery and patients treated with medical therapy.

The limitations of TEE include that it can be performed only at selected medical centers, it requires a specialized operator, and it cannot provide information about false lumen extension at the level of the abdominal aorta or about the involvement of abdominal aortic branch vessels $[35,36]$.

\section{Natural History}

\section{Aneurysm}

The natural history of a thoracic aneurysm is expansion, rupture, and death, with the greatest risk of rupture in larger aneurysms [2]. Factors affecting aneurysm expansion are increasing age, smoking, chronic obstructive pulmonary disease, and hypertension. The larger the aortic diameter, the higher the rate of aneurysm expansion, and in fact, an aneurysm $>50 \mathrm{~mm}$ expands faster than a smaller one. Also, the location of an aneurysm is crucial. Aneurysms located in the proximal descending aorta expand more rapidly than those located in the distal descending aorta [3, 4].

\section{Dissection}

Thoracic aortic type B dissection presents a severe prognosis in the acute phase; without any treatment it has a mortality rate of $33 \%$ at $24 \mathrm{~h}, 50 \%$ at $48 \mathrm{~h}$, and $75 \%$ at 2 weeks [16]. After the acute phase, uncomplicated type B dissection, which does not require interventional treatment, has a survival rate of $91 \%$ at 1 month and $89 \%$ at 1 year [37]. After 40 to 50 months, a thoracic aortic aneurysm develops in $20 \%$ to $30 \%$ of cases. This requires interventional management to prevent aortic rupture in $18 \%$ [38]. However, those patients who survive the acute phase have a good prognosis. Thus, any strategy that recommends 
intervention for all chronic stable type B dissections must ensure that the majority of survivors have an acceptable long-term outcome [39].

\section{Inclusion Criteria}

\section{Aneurysm}

In patients with a thoracic aneurysm that is $\leq 50 \mathrm{~mm}$ in diameter, no treatment is usually recommended, but regular-interval imaging follow-up must be performed (every 6 months). In these patients, moreover, close clinical surveillance is necessary, especially in order to monitor and maintain blood pressure at recommended values (systolic BAP level, $\leq 110 \mathrm{mmHg}$ ).

Repair of a thoracic aortic aneurysm should be considered when patients present with one of the following symptoms: chest discomfort, symptoms of surrounding organ compression (dyspnea, dysphagia, SOB, hemoptysis, etc.), and/or an aortic diameter $>55 \mathrm{~mm}$ [8]. Treatment is also indicated when the aneurysm diameter increases by more than $1 \mathrm{~cm}$ per year or when signs of aneurysm rupture are evident. Surgical therapy of descending aortic aneurysm with prosthetic graft repair is associated with a perioperative mortality rate ranging from $5 \%$ to $20 \%$, depending on the clinical conditions of the patient and the aneurysm diameter [13].

Aneurysms of the ascending aorta are generally treated with surgical reconstruction, while aneurysms of the descending aorta are addressed using either surgery or endovascular techniques. Endovascular treatment, based on the insertion of an endograft, represents a valid alternative to the conventional open repair with lower early morbidity and mortality rates. It is associated with a 30-day mortality rate ranging from $0 \%$ to $20 \%$ and a periprocedural stroke rate of from $0 \%$ to $7 \%[21,22]$.

\section{Dissection}

For type A dissection, surgery is still the treatment of choice. The surgical procedure should be performed emergently due to the possibility of serious ensuing complications related to rupture and/or involvement of the coronary arteries by the intimal flap.

In the case of type B dissection three treatment options should be considered:

- medical,

- surgical,

- endovascular.

Selection is based on the specific characteristics of the dissection and the clinical status of the patient.
Medical Therapy

Beta-blockers and ACE inhibitors in combination are considered the current treatment of choice in case of uncomplicated type B dissection, but in complicated cases with lower extremity malperfusion, visceral ischemia, and/ or renal failure, an immediate interventional treatment is necessary. Medical therapy provides good early-term results in uncomplicated dissection, with $85 \%$ of patients surviving the initial acute phase. The 30-day mortality rate is $10 \%$ for uncomplicated patients, versus $30 \%$ for those with complicated dissection [39]. Long-term results are poor, however, with a $50 \%$ mortality at 5 years and a high incidence of aneurysm formation (25\%) at 4 years [40].

In cases of complicated type B dissection (renal or abdominal ischemia) or unstable conditions (shock, severe uncontrollable hypertension), an invasive treatment should be considered.

\section{Surgery}

Open operative repair represents a valid therapeutic option in cases of acute type B dissection complicated by retrograde extension into the ascending aorta, Marfan syndrome, rupture, or involvement of vital organs. However, similar to the existing operative management considerations for thoracic aneurysms, surgery in the setting of complicated dissection is associated with a high incidence of paraplegia, prolonged hospital stay, and pulmonary complications.

\section{Endovascular Treatment}

Endograft placement is the new frontier for the treatment of type B dissection. The first report of endografting for an acute dissection was by Dake et al. in 1994 [1]. The rationale for endovascular therapy is to obliterate the false lumen and restore normal thoracic aortic anatomy. Stentgraft therapy will promote thrombosis of the false lumen and, in so doing, mitigate aneurysm development. Compared to open surgical repair, reports of this technique detail lower morbidity and mortality rates, especially for complications correlated to spinal cord ischemia [41].

Indications are as follows.

- Acute type B dissection in unstable patients when medical therapy cannot guarantee that blood pressure is controlled at a recommended low level (systolic BAP, $\leq 120 \mathrm{mmHg}$ ).

- Complicated acute type B dissection when the dissection involves an abdominal branch or the peripheral arteries, with consequent ischemia.

- Chronic type B dissection to avoid progressive dilatation of the aorta, with aneurysm formation and progressive risk of rupture. 
- Chronic type A dissection after surgical repair of the ascending aorta when the descending aortic false lumen is still patent and a progressive increase in its size/ volume is observed during follow-up.

Endovascular treatment is also recommended to solve ischemic branch complications correlated with the dissection.

A 30-day mortality rate of $10 \%$ is reported for uncomplicated type B dissection, while in cases of complicated dissection, mortality rates are higher: $20 \%$ at 2 days and $25 \%$ at 30 days [39]. Early results from different clinical series of stent-graft management in patients with acute and chronic type B dissection are encouraging [39, 42]. Obliteration of flow across the entry tear into the false lumen is achieved in $>90 \%$ of cases, with complete thrombosis of the proximal thoracic aortic false lumen over the length of the device in $80 \%$ to $95 \%$ [38].

\section{Endovascular Procedure}

Endovascular treatment of the thoracic aorta should be performed either in an operating room or in an angiosuite, in a sterile configuration, and with all the equipment necessary in case a surgical conversion is necessary. As the procedure requires angiograms to be performed in severe and/or compound oblique views in order to optionally evaluate the landing zones, it is crucial to use a state-ofthe-art fluoroscopy machine or dedicated new-generation $\mathrm{C}$-arm. Procedures can be performed with patients under local, epidural or general anaesthesia depending upon the patient's clinical conditions. General anesthesia should be selected, especially in unstable patients, to maintain appropriately low blood pressure levels.

The large diameter of the stent-graft device (22-25 Fr) requires a surgical cutdown to expose the common femoral artery, but recently TEVAR can also be performed with a completely percutaneous access with the use of percutaneous access closure devices. Avoidance of surgical femoral exposure may also result in shorter procedure times, consequent fewer local and systemic complications, and increased patient comfort. The technique is well tolerated by patients, with almost none of the postoperative discomfort typical of groin incision and with a rapid return to normal activities [43].

A graduated-marker pigtail catheter (4-5 Fr, $110 \mathrm{~cm}$ long) is introduced via the controlateral femoral site through a small introducer (4-5 Fr, $11 \mathrm{~cm}$ long). The stentgraft device is then advanced over a stiff guidewire in order to have enough support throughout the femoral and iliac systems. Through the pigtail catheter, several automated injections of contrast media are performed to correctly evaluate the morphology of the aorta. Then the device is advanced up to the desired position using fluoroscopy. If the stent-graft is deployed at the level of the aortic arch, the final aortogram should be performed with the device in its final position because the presence of a large and stiff device can modify a shift the arch morphology.

During the entire procedure, it is important to insure that the blood pressure never exceeds $100 \mathrm{mmHg}$, to avoid stent-graft misplacement.

\section{Technical Aspects}

\section{Landing Zone}

Thoracic aortic repair by endovascular stent-graft placement requires suitable proximal and distal landing zones for stable fixation and complete sealing of the endoprosthesis to the aortic wall. As the majority of the proximal fixation targets will be adjacent or within the aortic arch, this can be considered the Achilles' heel of TEVAR. The reasons are multiple and related mainly to the anatomy. In terms of endograft conformation to the underlying aortic wall, the arch is geometrically challenging and it contains critical branches. The knuckle of the arch refers to the area of the distal arch where the descending aorta takes its origin. This point represents a potential problem spot because presently available devices are unable to conform to such abruptly angled geometry, especially along the lesser curve, and/or a lack of fixation in this area can lead to a fatal disaster.

A problem arises when there is a short distance $(<20 \mathrm{~mm}$ ) between the origin of the LSA and an adjacent distal arch aneurysm or the primary entry tear of a type B dissection. Several options have been proposed to overcome this problem, such as prophylactic transposition of the LSA to the left common carotid artery (LCCA) or creation of a bypass graft between the left LCCA and LSA in order to provide sufficient blood flow to the arm [44].

Intentional occlusion of the LSA by thoracic stent-graft represents a valid alternative to the surgical procedures, especially in those patients with critical or emergent clinical conditions. In this case if symptoms, ischemic or neurological, develop, subsequent surgical revascularization of the LSA can be easily performed [45]. Total arch debranching is also possible, but it requires a sternotomy, with ascending aorta-based bypass grafts to all of the arch branches, followed by retrograde or antegrade endograft placement across the entire arch [46].

Alternatively, there are no easy management strategies to deal with a short distal neck above the celiac trunk. Intentional coverage of the celiac is not an innocuous procedure even in cases a coexisting normal superior mesenteric artery (SMA) capable of supporting an apparently normal network of collateral flow. Some authors report that pretreatment 
embolization of the celiac trunk is a reasonably safe alternative to create a longer landing zone at the level of the SMA. However, an accurate pretreatment evaluation is mandatory to evaluate the collateral flow at the level of the gastro-duodenal artery. In these cases, to guarantee patency of the SMA, a 4- to 5-Fr angiographic catheter is frequently placed within the SMA to serve as a reference marker during the thoracic device deployment.

\section{Device Insertion}

Patients undergoing TEVAR often have concomitant peripheral vascular disease involving the femoral and iliac arteries. Because the currently available devices employ relatively large delivery systems, their insertion can be challenging. Several techniques have been described to facilitate safe introduction of these device [47]. If a focal iliac lesion exists, a simple method to increase arterial caliber is to perform a PTA of the stenotic segment. However, dilation should be done very carefully, especially when the artery is calcified.

In cases where the femoral arteries are too small or where disease exists at the level of the external iliac arteries, the stent-graft device can be inserted through a common iliac artery exposed via a right or left lowerquadrant oblique incision. The device can be inserted either after direct arteriotomy or, alternatively, after anastomosing a vascular graft to the common iliac artery and creating a temporary conduit.

Alternatively, a right brachial approach can be used to insert the device if no other peripheral access is available, but this approach may be associated with neurological complications related to crossing the origin of the innominate trunk. A rare, but possible access that may be required in very unusual conditions is the common carotid artery.

Generally speaking, the right side provides a better angle for the insertion and delivery of the stent-graft device. It is advisable, however, to perform an accurate evaluation of the intracranial circulation to confirm the presence of adequate collateral flow via the anterior or posterior communicating arteries to avoid cerebral ischemia. Alternatively, the most direct approach for device introduction is to insert the delivery system via the abdominal aorta.

\section{Stent-Graft Dilation}

In cases of aortic aneurysm, gentle dilation of the stentgraft is performed at the level of the proximal and distal attachment sites to secure optimal wall apposition of the stent-graft. Dilation should be performed in a particular way with a rapid deflation of the balloon because balloon expansion is similar to aortic clamping and provokes a marked increase in blood pressure. Stent-graft dilation should be avoided in cases of dissection. A stent-graft's radial force is generally sufficient to obtain good aortic wall apposition and expansion of the true lumen. In fact, in these cases dilation may be associated with a progression of the dissection or rupture of the intimal flap. When more than one stent-graft device is implanted, dilation of the overlap zone between pieces is mandatory to ensure circumferential sealing between the different elements.

\section{Stent-Graft Selection}

Selection of the stent-graft (type, diameter, and length) is performed before the procedure, after accurate analysis of the diagnostic images. Selection of the correct diameter of the stent-graft can be difficult in dissection cases because the true lumen is only a fraction of the overall transaortic diameter and is rarely cylindrical in shape. Thus, several measurements should be performed along the dissected aorta, with special attention to the diameter of the nondissected aorta immediately proximal to the entry tear. Stent-graft selection is based on evaluation of the diameter of the healthy aorta just before the dissection [41]. Treatment of acute aortic dissections should be performed with minimal $(<2 \mathrm{~mm})$ or no oversizing using the nondissected midaortic arch as the target segment for measurement.

In cases of an aortic aneurysm, the stent-graft diameter is calculated on the basis of the proximal and distal neck diameters. In aneurysm cases, a device oversize factor, ranging between $20 \%$ to $30 \%$, is applied to select the most correct diameter of the endoprosthesis and to ensure a secure anchoring and a tight circumferential seal [48].

In cases of aortic dissection, another critical factor to decide is the length of the aorta to cover, in order to completely exclude the false lumen. Devices that are longer than the entry tear are often used, with resultant rapid formation of thrombi within the false lumen over the length of the device. The total length of the implant, however, must be weighed against the risk of spinal cord ischemia, which is increased with more extensive aortic coverage.

The complete exclusion of the aneurysm sac is based on the implantation of an endoprosthesis, at least $2 \mathrm{~cm}$ above and below the lesion. If more than one endoprosthesis is implanted, the overlap between two elements should be $>5 \mathrm{~cm}$ to avoid separation of the elements during the follow-up, especially in cases with very tortuous anatomy [49].

In the presence of a mismatch between the proximal and the distal landing zone diameters that exceeds $4 \mathrm{~mm}$, the procedure should be completed either using a tapered stentgraft or using two endoprosthesis of different diameters. The small endoprosthesis should be deployed first, and the larger device should be inserted into the smaller to facilitate good sealing. 
Selection of the ideal endograft for a particular case should be made on the basis of the morphological characteristics of the aorta in order to promote easy and accurate deployment, permanent fixation, and long durability. Endograft parameters that should be considered when making the choice are stent configuration, graft material, fixation mechanism, sizes, delivery system, tapered design, and radial force. Currently, different stent-grafts are commercially available on the European market:

- Gore TAG (W. L. Gore \& Associates, Flagstaff, AZ, USA)

- Valiant (Medtronic, Minneapolis, MN, USA)

- Zenith TX 2 (Cook Inc, Bloomington, IN, USA)

- Relay (Bolton Medical, Sunrise, FL, USA)

- EndoFit (LeMaitre Vascular, Burlington, MA, USA)

- E-vita (Jotec, Hechingen, Germany)

\section{TAG (W. L. Gore \& Associates)}

The TAG is formed from a nitinol stent skeleton lined with ePTFE (expanded-polytetrafluoroethylene) reinforced with a layer of ePTFE/fluorinated ethylene propylene (FEP). Both the proximal and the distal ends of the stent-graft have scalloped flares to facilitate conformity of the endograft to tortuous anatomy. A gold radiopaque marker at each end is located at the base of the flares. The TAG stentgraft is released from its middle portion toward each end simultaneously to reduce the deployment time. This is very important to avoid stent-graft misplacement, which can occur as a consequence of strong aortic flow forces that may distort and displace a partially deployed endograft.

The stent-graft is inserted via an introducer sheath that ranges from 20 to $24 \mathrm{Fr}$, in accordance with the stent-graft diameter. The TAG device is available in diameters ranging from 26 to $45 \mathrm{~mm}$ and in lengths of 10,15 , and $20 \mathrm{~cm}$.

\section{Valiant (Medtronic)}

The Valiant represents the latest evolution of the Talent stent-graft. It is made of a nitinol stent covered with polyester fabric. To improve deployment accuracy and technical ease, the long connecting bar of the Talent device has been removed, while columnar support has been optimized through stent spacing and the skeleton design. The proximal portion of the stent-graft is bare (free flow), while the distal end is covered. The metallic structure is supported by different rings of nitinol Z-stents connected to the grafts material with multiple polypropylene sutures.

Stent-graft diameters range from 22 to $46 \mathrm{~mm}$, with different lengths - 10,15 , and $22 \mathrm{~cm}$ - and with a delivery system of 22-25 Fr. The Valiant is available in both straight and tapered designs. The use of a special releasing system,
Xcelerant technology, allows a deployment that is more precise, more stable, and easier than that of the old Talent, even in cases of severe angulation of the aortic arch.

\section{Zenith TX2 (Cook Inc.)}

The Zenith TX2 is designed as a two-piece modular system, with one proximal and one distal component, although the implantation of a single piece may be sufficient for focal lesions. It is composed of stainless-steel Gianturco modified Z-stents covered with polyester (Dacron). At the ends of the endograft the stents are sewn inside the fabric, however, in the midportion they lie outside it. This design promotes fabric apposition to the aortic wall and fabric-tofabric interstent junctions. The proximal element presents a proximal bare end with protruding barbs with distal angulation to secure a better fixation to the aortic wall. The distal end of the proximal component is fully covered. The distal component presents a covered proximal portion and a distal bare stent with barbs.

Zenith endograft diameters range between 22 and $42 \mathrm{~mm}$ for the proximal component and from 28 to $42 \mathrm{~mm}$ for the distal element. Lengths range from 108 to $206 \mathrm{~mm}$ for the proximal element and from 127 to $207 \mathrm{~mm}$ for the distal one. The delivery system (20-22 Fr) is covered with a hydrophilic coating and is very flexible.

Recently, a new component was introduced on the market: the Zenith Dissection Endovascular Stent (TXD). It is a completely bare stent that is used to treat aortic dissection in conjunction with the TX2 proximal element in order to increase the true lumen diameter and reduce the risk of spinal cord ischemia.

\section{Relay (Bolton Medical)}

The Relay is composed of a polyester vascular graft fabric supported by a Nitinol stent and a spiral Nitinol wire that provides longitudinal stability. The stent-graft provides different levels of radial force over its length in order to create optimal wall apposition: the higher radial force is applied at both ends, while in the middle portion the radial force is less. A bare stent (free flow) is present at the proximal end of the endoprosthesis to better orient the angle of the proximal graft margin. The stent-graft is constrained within a flexible secondary sheath that is further constrained within an outer primary sheath. Once the device is advanced into the abdominal aorta, the secondary sheath is pushed out of the primary sheath. The flexibility of the secondary sheath allows easier navigation into the aortic arch and reduces friction during stent-graft deployment.

The delivery system ranges from 22 to $26 \mathrm{Fr}$ according to the diameter. The Relay is available in both straight and 
tapered designs, with diameters ranging from 22 to $46 \mathrm{~mm}$ and lengths up to $25 \mathrm{~cm}$.

\section{EndoFit (LeMaitre Vascular)}

The EndoFit is composed of an encapsulated body with two layers of laminated expanded polytetrafluoroethylene graft with nitinol Z-stent rings in between. Two different proximal end designs are available, with and without a bare stent.

The deployment system is based on a traditional pullback mechanism consisting of a 22- to 24-Fr device. Endofit graft diameters range from 34 to $42 \mathrm{~mm}$, with lengths up to $20 \mathrm{~cm}$.

\section{E-vita (Jotec)}

The E-vita is basically a nitinol stent covered with a polyester graft. An innovative release system for the graft provides full control and deployment accuracy even in cases with tortuous anatomy. Different proximal and distal configurations are available.

- The Straight Open design allows precise and safe positioning in the aortic arch.

- The Twin stent design features maximum radial force and an optimal sealing surface.

- The Straight Cut design features a circular distal terminus designed especially for type B dissections, whereas the Free Wire design allows a safe and secure anchoring mechanism while ensuring blood flow into the existing branch vessels.

Diameters range from 24 to $44 \mathrm{~mm}$, with varying lengths, up to $23 \mathrm{~cm}$. The size of the delivery system ranges from 20 to $24 \mathrm{Fr}$.

\section{Follow-Up}

As the procedure is still considered relatively "new," the adoption of a general protocol for accurate follow-up is necessary in order to critically evaluate any post stent-graft evolution of aortic morphology and the structure of the device. CTA is the current imaging method of choice because it provides all the critical information required to evaluate the aorta, its branches, the aneurysm sac morphology, and the presence of any endoleak. DSA is performed only in equivocal cases with ambiguous CT findings or when a complication occurs and conventional DSA is employed immediately prior to an endovascular reintervention.

A CTA follow-up exam is usually performed before the patient's discharge ( 3 to 5 days after the procedure), after 6 to 12 months, and yearly thereafter. Overall, aortic size, flow in the true and false lumens, diameter of the two lumens, endoleak, and characteristics of the stent-grafts are evaluated in each patient.

A postimplantation syndrome consisting of fever, mild leukocytosis, and elevated C-reactive protein was reported by Won et al. in 23 patients within 20 days of stent-graft placement for thoracic aortic dissections or aneurysms [50].

The initiation of false lumen thrombosis by sealing the primary entry tear induces both consolidation of the false lumen and remodeling of the aortic wall. Aortic stability results from both thrombosis of the false lumen and the endoprosthesis itself. Aortic remodeling consists of an active component (expansion of the true lumen) and a passive component (thrombus retraction in the false lumen) and mimics a natural healing process because a thrombosed false lumen is associated with a lower risk for future adverse events and better survival than a partially thrombosed or patent false channel.

\section{Left Subclavian Artery}

Typically, the use of commercially available stent-grafts requires a proximal neck length of at least $20 \mathrm{~mm}$ in the proximal descending aorta to achieve secure fixation and a tight seal between the graft and the aortic wall. If intentional occlusion of the LSA is planned to create a sufficiently long landing zone, accurate prestenting evaluation of both vertebral arteries with duplex ultrasound, DSA, CTA, or MRA is necessary to analyze their anatomy, patency, and continuity with the basilar artery.

In addition, the potential for ischemia of the left arm after the procedure may be predicted before stent-graft deployment by performing a 20-min test balloon occlusion of the proximal LSA. During the period of balloon occlusion, clinical monitoring of left arm symptoms is performed to assess the status of the collateral circulation. However, if there is documented normal flow in both vertebral arteries and intact anatomical connections to the basilar artery, a preinterventional balloon occlusion test may be avoided.

Several papers document the safety of intentional occlusion of the LSA by an aortic stent-graft without prophylactic surgical transposition [44, 49]. Alternatively, it is possible to limit any ischemic complication associated with LSA exclusion by adjunctive operative strategies of surgical transposition of the LSA to the left common carotid artery or of the left common carotid artery to the LSA surgical bypass. These interventions must be performed prior to stent-graft coverage of the LSA in those patients with a documented incomplete circle of Willis that compromises collateral flow, critical stenosis of the vertebral arteries, anatomical variant of the right subclavian artery (lusorian subclavian artery), or compromised collateral circulation to the left arm from variant anatomy such as an 
independent left vertebral artery origin from the arch or a previous aortocoronary bypass performed with the left internal mammary artery.

Recently, a new strategy to manage the LSA has been introduced with the development of a branched stent-graft designed to maintain the normal antegrade flow into the LSA [51, 52].

\section{Complications}

Endovascular treatment of the thoracic aortic pathologies has been firmly established as a valid alternative to surgery. As this treatment becomes more and more widespread, procedural-related complications are more widely recognized, although the majority of these treatment-related problems can be managed with catheter-based interventions. Only critical conditions, such as stent-graft infection and migration, may ultimately require endograft removal followed by conventional open surgery repair.

\section{Spinal Cord Ischemia}

One major problem related to type $\mathrm{B}$ dissection repair is spinal cord ischemia, especially after surgery [23]. The effect of endoluminal repair on the spinal cord is still uncertain but the absence of aortic clamping, which may cause left-sided heart failure and spinal cord ischemia, may reduce the incidence of paraplegia (in many series it is $<3 \%$ ) relative to open surgery $[17,37]$.

TEVAR is generally associated with a $3 \%$ to $6 \%$ frequency of spinal cord ischemia secondary to the interruption of multiple-branch vessels that provide spinal cord perfusion. Sacrificing critical intercostals can lead to immediate paraplegia but the multiple collateral pathways between the aorta and the spinal cord allow the maintenance of good perfusion in many cases, even if some intercostals are sacrificed. Factors that influence the development of spinal cord ischemia include prior abdominal aortic repair, length of thoracic aortic coverage, hypogastric artery interruption, subclavian artery coverage, emergency repair, and hypotension.

To reduce the risk of spinal cord ischemia during surgical procedures, several interventions have been suggested, such as cerebrospinal fluid (CSF) drainage, intercostal artery reimplantation, maintenance of normotension, and hypothermia. CSF drainage via a lumbar drain can be easily performed and is used to maintain the pressure of the cerebrospinal fluid at $\leq 15 \mathrm{mmHg}$, in concert with keeping the mean arterial blood pressure at $\geq 90 \mathrm{mmHg}$. Initial results suggest that this policy is applicable to patients treated with endovascular therapy, particularly for patients who have undergone a previous abdominal aortic procedure or in whom a long stent-graft must be implanted.

Cheung et al. reported that no single intercostal arterial pair at any vertebral level is absolutely necessary for spinal cord integrity. Moreover, they noted that the risk of paraplegia increases if more than 10 intercostal pairs are sacrificed [53].

Endoleak

Endoleak represents the most common complication following the endovascular treatment of aortic pathologies, with a rate ranging from $4 \%$ to $24 \%$ [48]. Leakage is classified according to the site of its origin at the proximal, distal, or mid graft. Proximal or distal endoleak is due to incomplete fixation of the stent-graft to the aortic wall neck(s) (type I), while a leak at the midgraft level is consequent to retrograde blood flow via an aortic branch (type II) or graft defects (type IV). Endoleaks can also originate from an incompetent overlap seal between stent-grafts (type III) when multiple devices are implanted [40, 41].

The prognosis for type I endoleak is generally poor and aggressive treatment is mandatory. Endovascular or surgical intervention is recommended when a type I endoleak is documented more than 2-4 weeks after stent-graft implantation. Type I endoleak at the level of the proximal neck represents a very dangerous event, with continuous direct arterial pressurization of the false lumen. In these cases an immediate intervention is mandatory, with the deployment of one or more endograft cuffs.

Type II endoleak is associated with residual blood flow into the aneurysmatic sac or the false lumen from patent intercostals arteries, bronchial arteries, or patent LSA. In cases of TAA, if no documented enlargement of the sac is observed, regular-interval follow-up imaging surveillance is the most prudent course of action. In the case of sac enlargement or persistent patency of the false lumen, percutaneous treatment with selective catheter embolization is suggested and easily performed.

Type III endoleak, secondary to the disconnection of different stent-graft elements, requires immediate treatment to avoid severe complications due to continuous flow within the aneurysm or the false lumen. In these cases, endovascular therapy can be performed with the insertion of a new endoprosthesis inside the previous ones. In more complex cases, surgical explantation is the best solution.

Type IV endoleak is related to the porosity or damage of the graft material.

\section{Retrograde Aortic Dissection}

Retrograde aortic dissection represents a catastrophic sequela more evident during treatment of type B dissection. 
This complication is associated with the use of an especially stiff device, especially in cases where there is a severe angle of the aortic arch. In fact, if insufficient support is provided by the guidewire during advancement, the device can be pushed against the greater curvature of the aortic arch, increasing the risk of wall damage. Retrograde aortic dissection, involving the aortic arch and the ascending aorta, can also be caused by an endograft with excessive radial force that may cause an intimal tear within the proximal landing zone. Sometimes, the aggressive or inappropriate manipulation of catheters and wires can be responsible for a new intimal tear that facilitates a retrograde dissection. Several studies have reported retrograde dissection involving the aortic arch and ascending aorta after stent-graft deployment [54]. In these cases, the new intimal tear can be managed either with deployment of an additional stent-graft over it or with surgery.

\section{Neurological Injury}

The etiology of intracranial injuries associated with endograft placement is multifactorial. Different authors indicate neurological complications secondary to LSA exclusion, as well as stent-graft and wire manipulations at the level of the arch [55]. This condition seems to be more frequent in patients with atherosclerotic aneurysms. Moreover, Feezor et al. reported that $56 \%$ of individuals with stroke during TEVAR had documented intraoperative hypotension with a systolic blood pressure $<80 \mathrm{mmHg}$ [55].

\section{Conclusions}

Endovascular treatment of a variety of aortic pathologies is considered a valid alternative to open surgery, with reduced rates of morbidity and mortality relative to conventional operative repair. This less invasive method for treating these potentially catastrophic aortic lesions has created great enthusiasm, however, careful and sound considerations regarding an individual patient's anatomic suitability, clinical appropriateness, and institutional experience should always be carefully judged. An important debate regarding the long-term effectiveness of thoracic aortic stent-grafting is ongoing among researchers interested in defining the legitimate role of this therapy in the management of thoracic aortic pathologies.

As technology and available devices improve day by day, the number of patients undergoing endovascular repair will certainly increase. At the same time, it is anticipated that the limitations associated with this technology will decrease as delivery systems become smaller in size, and interventionists gain more experience determining optimal patient selection.
Open Access This article is distributed under the terms of the Creative Commons Attribution Noncommercial License which permits any noncommercial use, distribution, and reproduction in any medium, provided the original author(s) and source are credited.

\section{References}

1. Dake MD, Miller C, Semba CP et al (1994) Transluminal placement of endovascular stent grafts for the treatment of descending thoracic aortic aneurysms. N Engl J Med 331:1729-1734

2. Crawford ES, De Natale RW (1986) Thoracoabdominal aortic aneurysm: observations regarding the natural course of the disease. J Vasc Surg 3:578-582

3. Juvenon T, Ergin MA, Galla JD et al (1997) Prospective study of the natural history of thoracic aortic aneurysms. Ann Thorac Surg 63:1533-1545

4. Juvonem T, Ergin MA, Galla JD et al (1997) Prospective study of the natural history of thoracic aortic aneurysms. Ann Thorac Surg 63:1533-1545

5. Crawford ES, Crawford JL, Safi HJ et al (1986) Thoracoabdominal aortic aneurysms: preoperative and intraoperative factors determining immediate and long-term results of operations in 605 patients. J Vasc Surg 3:389-404

6. Shimada I, Rooney S, Farnetti PA et al (1999) Prediction of thoracic aortic aneurysm expansion: validation of formulae describing growth. Ann Thorac Surg 67:1968-1970

7. Strachan DP (1991) Predictors of death from aortic aneurysm among middle-aged men: the Whitehall study. Br J Surg 78:401-404

8. Coady MA, Rizzo JA, Hammond GL et al (1997) What is the appropriate size criterion for resection of thoracic aortic aneurysms? J Thorac Cardiovasc Surg 113:476-491

9. Cambria RP, Brewster DC, Lauterbach SR et al (2002) Evolving experience with thoracic aortic stent-graft repair. J Vasc Surg 35:1129-1136

10. Griepp RB, Ergin MA, Lansman SL et al (1991) The natural history of thoracic aortic aneurysms. Semin Thor Cardiovasc Surg 3:258-265

11. Criado FJ, Abul-Khoudoud OR, Domer GS et al (2005) Endovascular repair of the thoracic aorta: lesson learned. Ann Thorac Surg 80:857-863

12. Finkbohner R, Johnston D, Crawford ES et al (1995) Marfan syndrome: long-term survival and complications after aortic aneurysm repair. Circulation 91:728-733

13. Svensson LG, Crawford ES, Hess KR et al (1990) Dissection of the aorta and dissecting aortic aneurysms: improving early and long-term surgical results. Circulation 82(Suppl 5):IV24-IV38

14. Crawford ES, Svensson LG, Coselli JS et al (1988) Aortic dissection and dissecting aortic aneurysms. Ann Surg 208:254

15. Svensson LG, Crawford ES, Hess KR et al (1990) Dissection of the aorta and dissecting aneurysms improving early and long term results. Circulation 82(Suppl IV):24

16. Tsai TT, Fattori R, Trimarchi $S$ et al (2006) Long-term survival in patients presenting with Type B acute aortic dissection: insights from the international registry of acute aortic dissection. Circulation 114:2226-2231

17. Nienaber CA, Rehders TC, Ince H (2006) Interventional strategies for treatment of aortic dissection. J Cardiovasc Surg 47:487-496

18. Daily PO, Trueblood HW, Stinson EB et al (1970) Management of acute aortic dissections. Ann Thorac Surg 10:237-247

19. Nienaber CA, Eagle MA (2003) Aortic dissection: new frontiers in diagnosis and management. Circulation 108:628-635

20. Fuster V, Halperin JL (1994) Aortic dissection: A medical perspective. J Cardiac Surg 9:713-728 
21. Fattori R, Napoli G, Lovato L et al (2003) Descending thoracic aortic diseases: stent-graft repair. Radiology 229:176-183

22. Mitchell RS, Dake MD, Sembra CP et al (1996) Endovascular stent-graft repair of thoracic aortic aneurysms. J Thorac Cardiovasc Surg 111(5):1054-1062

23. Coselli JS (1996) Thoracoabdominal aortic aneurysms. Ann Thorac Surg 61(1):269-270

24. Dake MD, Kato N, Mitchell RS et al (1999) Endovascular stentgraft placement for the treatment of acute aortic dissection. $\mathrm{N}$ Engl J Med 340:1546-11552

25. Crawford ES, Svensson LG, Coselli JS et al (1988) Aortic dissection and dissecting aortic aneurysms. Ann Surg 208:254

26. Cambria RP, Brewster DC, Moncure AC et al (1988) Spontaneous aortic dissection in the presence of coexistent or previously repaired atherosclerotic aortic aneurysm. Ann Surg 208:619-624

27. Cambria RP, Brewster DC, Gertler J et al (1988) Vascular complications associated with spontaneous aoritc dissection. J Vasc Surg 7:199-209

28. Bortone AS, De Cillis E, D'Agostino D et al (2004) Stent-graft treatment of thoracic aortic disease. Surg Technol Int 12:189-193

29. Cigarroa JE, Isselbacher EM, De Sanctis RW et al (1993) Diagnosis imaging in the evaluatation of suspected aortic dissection. Old standards and new directions. N Engl J Med 328:35

30. Quint LE, Francis IR, Williams DM et al (1997) Evaluation of thoracic aortic disease with the use of helical CT and multiplanar reconstructions: comparison with surgical findings. Radiology 202:183-193

31. Koktzoglou I, Kirplani A, Carroll TJ et al (2007) Dark-blood MRI of the thoracic aorta with 3D diffusion prepared steady-state free precession: Initial clinical evaluation. Am J Radiol 189:966-972

32. Zhang J, Fletcher JG, Vrtiska TJ et al (2007) Large-vessel distensibility measurement with electrocardiographically gated multidetector CT: phantom study and initial experience. Radiology 245:258-266

33. Pannu HK, Jacobs JE, Lai $S$ et al (2006) Gated cardiac imaging of the aortic valve on 64-slice multidetector computerized tomography, preliminary observation. J Comput Assist Tomogr 30:443-446

34. Lee VS, Hecht EM, Taouli B (2007) Body and cardiovascular MR imaging at 3.0 T. Radiology 244:692-705

35. Chirillo F, Cavallini C, Longhini C et al (1994) Comparative diagnostic value of transesopahgeal echocardiography and retrograde aortography in the evaluatation of thoracic aortic dissection. Am J Cardiol 74:590-595

36. Nienaber CA, Von Kodolitsch Y, Volkmar N et al (1993) The diagnosis of thoracic aortic dissection by noninvasive imaging procedures. N Engl J Med 328:1-9

37. Genoni M, Paul M, Tavakoli R et al (2002) Predictors of complications in acute type B dissection. Eur J Cardiothor Surg 22:59-63

38. Grabenwogen M, Fleck T, Czerny M et al (2003) Endovascular stent graft placement in patients with acute thoracic aortic syndromes. Eur J Cardiothorac Surg 23:788-793

39. Winnerkvist A, Lockowandt U, Rasmussen E et al (2006) A prospective study of medically treated acute type B aortic dissection. Eur J Vasc Endovasc Surg 32:349-355
40. Nienaber CA, Zanetti S, Barbieri B et al (2005) INvestigation of STEnt grafts in patients with type B Aortic Dissection: design of the INSTEAD trial-a prospective, multicenter European randomized trial. Am Heart J 149:592-599

41. Fattori R, Nienaber CA, Rousseau H et al (2006) Results of endovascular repair of the thoracic aorta with the Talent thoracic stent-graft: the Talent thoracic retrospective registry. J Thorac Cardiovasc Surg 132:332-332

42. Umana JP, Lai DT, Mitchell RS et al (2002) Is medical therapy still the optimal treatment strategy for patients with acute type B aortic dissections? J Thorac Cardiovasc Surg 124:896-910

43. Lee WA, Brown MP, Nelson PR et al (2008) Midterm outcomes of femoral arteries after percutaneous endovascular aortic repair using the Perclose technique. J Vasc Surg 47:919-923

44. Gorich J, Asquan Y, Seifarth H et al (2002) Initial experience with intentional stent-graft coverage of the subclavian artery during endovascular thoracic aortic repairs. J Endovasc Ther 9(Suppl 2):II39-II43

45. Chuter TA, Schneider DB, Reilly LM et al (2003) Modular branched stent graft for endovascular repair of aortic arch aneurysm and dissection. J Vasc Surg 38(4):859-863

46. Vos AW, Linsen MA, Wisselink W et al (2004) Endovascular grafting of complex aortic aneurysms with a modular side branch stent-graft system in porcine model. Eur J Vasc Endovasc Surg 27(5):492-497

47. Criado FJ (2007) Iliac arterial conduits for endovascular access: technical considerations. J Endovasc Ther 14:347-351

48. Nienaber CA, Kische S, Ince H (2007) Thoracic aortic stent-graft devices: problems, failure modes, and applicability. Semin Vasc Surg 20:81-89

49. Hausegger KA, Oberwalder P, Tiesenhausen T et al (2001) Intentional left subclavian artery occlusion by aortic stent-grafts without surgical transposition. J Endovasc Ther 8(5):472-476

50. Won JY, Lee DY, Shim WH et al (2001) Elective endovascular treatment of descending thoracic aortic aneurysms and chronic dissections with stent-grafts. J Vasc Interv Radiol 12:575-582

51. McWilliams RG, Murphy M, Hartley D et al (2004) In situ stentgraft fenestration to preserve the left subclavian artery. J Endovasc Ther 11:170-174

52. Saito N, Kimura T, Odashiro K et al (2005) Feasibility of the Inoue single- branched stent-graft implantation for thoracic aortic aneurysm or dissection involving the left subclavian artery: short to medium term results in 17 patients. J Vasc Surg 41:206-212

53. Cheung AT, Pochettino A, McGarvey ML et al (2005) Strategies to manage paraplegia risk after endovascular stent repair of descending thoracic aortic aneurysms. Ann Thor Cardiovasc Surg 80:1280-1289

54. Fanelli F, Marcelli G, Salvatori FM et al (2003) Type A aortic dissection Developing during endovascular treatment of an acute type B dissection. J Endovasc Ther 10:254-259

55. Feezor RJ, Martin TD, Hess PJ et al (2007) Risk factors for perioperative stroke during thoracic endovascular aortic repairs (TEVAR). J Endovasc Ther 14:568-573 